\title{
Recensión del libro "La vie en réseau. Dynamique des relations sociales" de Claire Bidart, Alain Degenne y Michel Grossetti
}

\author{
Ainhoa de Federico - Universidad de Toulouse ${ }^{1}$
}

\section{Resumen}

Este libro es una contribución fundamental al estudio de la dinámica de las redes personales. Comienza definiendo qué es una relación, qué es una red y cómo ambas se articulan entre sí y con los círculos sociales más estructurados en que emergen. A partir de un marco teórico claro, permite describir y comprender los distintos tipos de dinámicas implicadas en las relaciones y las redes, en relación con su historia, su articulación con el mundo social, los eventos biográficos, las trayectorias de vida y la estructura social.

Palabras clave: Teoría de redes sociales, dinámicas relacionales, dinámica de las redes personales, trayectoria vital y redes, estructura social y redes.

\begin{abstract}
Tis book is a fundamental contribution to the stydy of personal networks. It begins by defining what is a social relation, what is a social network and how they articulate among them and with the more structured social circles where they emerge. Relying on a very clear theoretical frame it allows to describe and understand the different types of dynamics implied in relations and networks in relationship with their history, their articulation with the social world, the biographic events, life trajectories and social structure.
\end{abstract}

Key words: Social network theory, relational dynamics, personal network dynamics, life trajectory and networks, social structure and networks.

Disipemos antes de nada un posible malentendido. El libro La vie en réseaux. Dynamique des relations sociales. (La vida en red. Dinámicas de las relaciones sociales) publicado en París, Presses Universitaires de France (335 pag.) no trata sobre redes sociales en internet (tipo Facebook, Tuenti o Linkedin), sino de las redes de relaciones interpersonales que son hechos sociales a la vez subyacentes, mucho más antiguos, más complejos, estudiados por las ciencias sociales desde los años 50 ' y la necesaria base sobre la que las "redes sociales" en las NTIC se apoyan para desarrollarse.

Este libro trata de dar una visión de conjunto de las relaciones personales como fenómenos sociales emergentes $y$, sobretodo, de su dinámica en la escala fina de la propia relación y de las redes personales en las que se encuentran insertos los individuos. Trata también, al mismo tiempo, de romper una lanza por una

\footnotetext{
${ }^{1}$ Enviar correspondencia a: Ainhoa de Federico ainhoa.defederico@univ-tlse2.fr
} 
sociología relacional, es decir una sociología que toma las relaciones como objeto de estudio central pero que al mismo tiempo es capaz de situarlas y articularlas con otras dimensiones colectivas de la vida social. Se trata también, por lo tanto, de un libro que realiza una contribución a la teoría sociológica general al ser capaz de articular la sociología relacional con otros niveles de estructuración de los mundos sociales.

Se puede considerar ya un libro imprescindible dentro de la sociología de las redes sociales o del análisis de redes sociales. El análisis de redes sociales, desarrollado inicialmente en América del Norte y posteriormente en Europa, se centró en un principio en la descripción de las estructuras y sólo más tarde en su dinámica, pero principalmente en las llamadas "redes completas" que usualmente - aunque hay muy honrosas excepciones - se centran en estudios de caso de universos relativamente circunscritos y difícilmente generalizables. La contribución central del libro no es únicamente haber realizado una síntesis organizada y estructurada de los conocimientos acumulados sobre la dinámica de las redes personales dentro de esta tradición de investigación, sino también sus numerosas contribuciones originales a la comprensión de los distintos niveles de dinámica en las redes personales, así como su articulación con las trayectorias biográficas y la articulación con otros conceptos centrales de la sociología y de la estructura social. Las contribuciones originales han sido posibles principalmente gracias al análisis de dos investigaciones empíricas originales dedicadas a describir en detalle las redes personales realizadas por los autores: el llamado "Panel de Caén" iniciado con 87 jóvenes de secundaria en 1995 y que se ha repetido cada 3 años cuatro veces más y una encuesta puntual realizada en Toulouse con 399 habitantes de la aglomeración y alrededores.

A la claridad conceptual y a la estructura rigurosa del libro, que facilita enormemente la comprensión, se añaden dos cualidades sugestivas y estimulantes: la combinación de datos estadísticamente representativos con extractos de entrevistas que permiten ilustrar las redes y sus dinámicas ofreciendo un rostro humano a los perfiles típicos de que se habla; también el hecho de que se aborden un cierto número de situaciones específicas, distantes del "ciudadano medio", pero de gran interés sociológico y a veces de gran impacto social (algunos ejemplos de dichas situaciones específicas son, por ejemplo, las dinámicas relacionales de los migrantes o de las personas que, descubriéndose homosexuales, deciden salir del armario). 
El libro está dividido en tres partes. La primera parte define con claridad los conceptos y los objetos de estudio: las relaciones y las redes. Las relaciones sociales se definen a partir de las distintas dimensiones implicadas: la interacción en el tiempo, el conocimiento, la implicación emocional, y el móvil del vínculo, que son los elementos indispensables para considerer que dicho fenómeno existe. También se discuten algunas de sus características como la fuerza del vínculo y su polivalencia. Después se sitúa la especificidad de la relación como forma social en relación con otras de sus estructuras: las redes y los círculos sociales, y se estudian las relaciones entre ellos. Se aborda también la homogeneidad de las relaciones (homofilia) y el hecho de que éstas constituyen recursos. Finalmente, a partir de una propuesta conceptual de Alexis Ferrand, se presentan distintos tipos de regulación que intervienen en las relaciones sociales. Las redes constituyen conjuntos articulados de relaciones sociales. Se examinan por lo tanto sus características: composición, densidad, homogeneidad y dispersión. A continuación se muestra la variedad de estructuras de conjunto posibles en las redes según los entornos sociales que impliquen, de este modo se puede trazar una cierta cartografía social dinámica de los modos de circulación y un anclaje dentro de distintos universos sociales.

La segunda parte, está destinada al estudio de distintos niveles de dinámicas de las relaciones y las redes. Para empezar los autores describen el marco teórico en el que se apoyan para analizar los procesos presentados en este capítulo y los siguientes. Este permite dar cuenta de las interacciones entre los círculos sociales, las relaciones y las redes, y de tener en mente que los unos no existen sin los otros. Se exploran después sucesivamente tres grandes tipos de contextos de emergencia de las relaciones: su construcción dentro de un círculo, las interacciones en torno a actividades u objetivos comunes o la extensión de la red por conocidos comunes. Una vez que las relaciones se crean adquieren cierta autonomía respecto a su contexto de emergencia. Dentro de los entornos sociales, distintas dinámicas relacionales hacen que las relaciones se transformen a lo largo del tiempo según cómo se articulen con los contextos y las redes. Los movimientos de polivalencia / especialización, singularización / imbricación y conexión / disociación se estudian en relación con la duración de las relaciones y también con respecto a las trayectorias biográficas de los encuestados. Sigue un capítulo en que se examinan los factores que intervienen en la muerte de las relaciones o por el contrario, en su durabilidad. Se estudia igualmente su articulación con la estructura de la red: la muerte de las relaciones afecta a ésta, pero también la estructura interviene en la solidez de los lazos. Se combinan aquí por lo tanto una visión 
global de dichos movimientos así como el examen de lógicas más finas de la continuidad o el fin de las relaciones. A continuación se aborda el estudio de las dinámicas de las redes. Se examina cómo éstas se constituyen a lo largo del tiempo, cómo se articulan las relaciones emergentes en distintos periodos de la vida y cómo cambia la estructura de la red a lo largo del tiempo. Observando los procesos de disociación o de agregación de las relaciones, de imbricación o singularización respecto a los contextos sociales, los autores identifican algunos procesos típicos de evolución de las redes sociales. En particular estas dinámicas se estudian en relación con distintos eventos biográficos. La sección termina con las transformaciones típicas de las redes a lo largo de las trayectorias de vida y sus periodos de reconfiguración de las redes. La familia de origen cede su lugar a las relaciones de afinidad, y éstas se van renovando y cambiando hasta que sufren el impacto de la jubilación. Se examinan con detalle en particular los eventos implicados en la transición "ordinaria" a la vida adulta y algunos eventos menos frecuentes.

Esta segunda parte constituye la contribución científica original más importante, que sitúa a este libro entre los libros de investigación punteros en análisis de redes personales. La tercera parte vincula las dinámicas de las redes con la estructuración del mundo social, situándolo por lo tanto al mismo tiempo como un libro que efectúa una contribución a teoría sociológica general y como manual.

En ésta primeramente se relacionan las redes sociales con el espacio geográfico. ¿Hasta qué punto, en la era de las NTIC, las relaciones se ven desvinculadas de la distancia? ¿Dónde viven las personas más cercanas afectivamente? ¿Cuáles son los efectos de las evoluciones urbanas en las estructuras relacionales? Y la movilidad ¿cómo afecta a las relaciones? Este capítulo aborda la dimensión espacial de la proximidad relacional para redescubrir la importancia de la aglomeración urbana como espacio principal del desarrollo relacional. También recuerda la capacidad de las relaciones más cercanas para sobrevivir a pesar de la distancia $Y$ descubre la capacidad de las redes para conservar su estructura después de una movilidad, a pesar de los cambios importantes en la composición de la red.

Después de la distancia geográfica se examina la relación de las redes sociales con la distancia social y se aborda el juego de afinidades que favorece más o menos, según el contexto, al establecimiento de relaciones con personas de características similares. El primer ejemplo clásico abordado, de la formación de las parejas, llamado "homogamia" tiene su equivalente para las relaciones generales, la "homofilia". Las desigualdades sociales dejan su huella en las características de las 
redes, en su articulación con los círculos sociales y en la evolución de las redes que las componen. Incluso las afinidades electivas que quisiéramos libres del peso de la estructura y las categorías sociales se ven en parte sometidas a ellas. Así pues, se describe una segregación suave basada en el hecho de que las relaciones libremente escogidas pueden paradójicamente contribuir a fragmentar el mundo social.

A continuación se presentan las relaciones y las redes como recursos. Estas no son sólo la expresión de la sociabilidad y el placer de compartir; también tienen una dimensión instrumental o de utilidad ya que bajo ciertas condiciones pueden dar acceso a recursos. Las relaciones son el soporte de numerosos intercambios de servicios y de ayuda mutua. Se examinan por lo tanto aquí las relaciones como recursos o como medio para obtenerlos (p.e. lograr un trabajo). Entonces, si las relaciones pueden ser recursos, ¿cómo se distribuyen? ¿se puede hablar de desigualdades relacionales como se puede hablar de otras desigualdades sociales? Y ¿de qué factores dependen? Como en otros aspectos de la vida social, en materia de relaciones los factores sociales influyen en el "capital relacional". Según el medio social, los estudios, la profesión, los eventos biográficos, así como las elecciones personales, las redes obtenidas serán diferentes. Se estudian aquí por lo tanto las desigualdades relacionales así como los procesos que las producen y las amplifican.

El libro termina con la pregunta abierta sobre los márgenes de libertad que dan las redes dada su inscripción en otras estructuras sociales: ¿refuerzan las desigualdades o permiten otros espacios intersticiales de solidaridad? En cualquier caso los procesos relacionales como fenómeno de rango medio deben estudiarse conjuntamente con los otros niveles de estructuración más clásicos del mundo social.

Esta obra realiza de este modo una gran contribución, tanto en términos de investigación científica como por su inscripción en la teoría sociológica. A pesar de ello, hay cuatro áreas que me parecen pistas posibles de desarrollo para la investigación futura sobre la dinámica de las relaciones y las redes personales. Aunque no están completamente ausentes de la redacción del libro y los propios autores indican a veces que son fenómenos que quedan por explorar más ampliamente, me parece importante resaltarlos. 1. Las formas relacionales y sus contenidos son situacionales y por lo tanto dependientes del contexto histórico. Aunque este aspecto quede fuera de la disciplina sociológica, una visión histórica sobre la evolución de los distintos tipos y formas de relaciones (de amistad, de parentesco, otras) añadiría una perspectiva interesante para comprender hasta qué 
punto hablamos de formas socialmente construidas. 2. Si el análisis histórico permite una perspectiva diacrónica sobre lo construido sobre la relación social y sus dinámicas, las comparaciones internacionales permitirían el punto de vista sincrónico sobre la cuestión. Si ser madre, amigo o colega no significa lo mismo en diferentes sociedades, la dinámica de dichas relaciones en distintos momentos biográficos y en distintas posiciones sociales probablemente también será diferente. Aunque los autores se apoyan en una gran cantidad de encuestas disponibles en Francia, Europa y América del Norte, esta cuestión merecería un análisis más detallado. Para poder realizarlo serían necesarias encuestas comparativas internacionales con un grado de descripción fino de las relaciones y las redes personales y dichas encuestas son prácticamente inexistentes. A veces la curiosidad científica se ve limitada por las contingencias de la realidad. 3. Si la dinámica de las relaciones y las redes se estudia en relación con la estructura espacial y la movilidad geográfica, el libro se centra principalmente en la movilidad intranacional. La movilidad inter-nacional implica distintos procesos específicos (cruzar las fronteras implica mayores cambios institucionales, lingüísticos, económicos, de posición en la estructura social, de costumbres etc.) que probablemente alteran las dinámicas relacionales y las de las redes de formas diferentes. Cabe destacar en este sentido la gran vitalidad de las investigaciones de las redes personales en los procesos migratorios realizados por distintos investigadores diseminados por todo el territorio español (Barcelona, Huelva, Málaga, Sevilla...) y bien conocidas a nivel europeo. 4 Por último, este libro trata, como la mayoría de las investigaciones en análisis de redes sociales, sobre relaciones positivas. Ya es una contribución importante que se considere su emergencia, evolución, latencia y a veces desaparición. Pero queda fuera del enfoque del libro considerar las relaciones negativas, nocivas, hostiles, dañinas o los efectos indirectamente nocivos de relaciones explicitas o aparentemente positivas. Se trata esta de una línea de investigación nueva y emergente como lo demuestran sendas jornadas de investigación realizadas en la primavera del 2012 en Toulouse y Budapest.

A pesar de estas puntualizaciones el libro se ha convertido desde su publicación en un clásico indispensable tanto para el estudiante que quiere introducirse en el análisis de redes sociales como para el investigador que quiera profundizar en la comprensión de las dinámicas de las redes personales. No solo recomiendo su lectura sino que me hubiese encantado participar en su escritura. 